\title{
The role of evolution in shaping ecological networks
}

by Segar, S.T., Fayle, T.M., Srivastava, D.S., Lewinsohn, T.M., Lewis, O.T., Novotny, V., Kitching, R.L. and Maunsell, S.C.

Copyright, publisher and additional Information: This is the author accepted manuscript. The final published version (version of record) is available online via Elsevier.

This version is made available under the CC-BY-ND-NC licence:

https://creativecommons.org/licenses/by-nc-nd/4.0/legalcode

Please refer to any applicable terms of use of the publisher

DOI: https://doi.org/10.1016/j.tree.2020.01.004

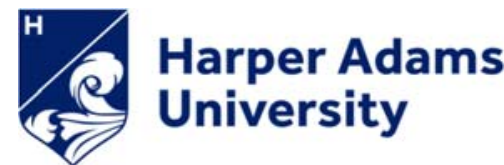

Segar, S.T., Fayle, T.M., Srivastava, D.S., Lewinsohn, T.M., Lewis, O.T., Novotny, V., Kitching, R.L. and Maunsell, S.C. 2020. The Role of Evolution in Shaping Ecological Networks. Trends in Ecology \& Evolution.

12 February 2020 


\section{The role of evolution in shaping ecological networks}

Simon T. Segar ${ }^{1,2,3^{*}}$, Tom M. Fayle ${ }^{1,2,4}$, Diane S. Srivastava ${ }^{5}$, Thomas M. Lewinsohn ${ }^{6,7}$, 5 Owen T. Lewis ${ }^{8}$, Vojtech Novotny, ${ }^{1,2}$, Roger L. Kitching ${ }^{9}$, Sarah C. Maunsell ${ }^{10}$

${ }^{1}$ Biology Centre, Czech Academy of Sciences, Institute of Entomology, Branisovska 1760, 37005 Ceske Budejovice, Czech Republic,

${ }^{2}$ University of South Bohemia in Ceske Budejovice, Faculty of Science, Branisovska 1760, 37005 Ceske Budejovice, Czech Republic,

$10{ }^{3}$ Department of Crop and Environment Sciences, Harper Adams University, Newport, Shropshire, TF10 8NB, UK

${ }^{4}$ Institute for Tropical Biology and Conservation, Universiti Malaysia Sabah, Kota Kinabalu, Sabah, Malaysia,

${ }^{5}$ Dept of Zoology \& Biodiversity Research Centre, University of British Columbia 6270

15 University Blvd Vancouver BC Canada V6T 1Z4,

${ }^{6}$ Depto Biologia Animal, Instituto de Biologia, University of Campinas, Campinas 13083870, São Paulo, Brazil,

${ }^{7}$ Wissenschaftskolleg zu Berlin, Berlin 14193, Germany,

${ }^{8}$ Department of Zoology, South Parks Road, Oxford, OX1 3PS, UK,

$20{ }^{9}$ Environmental Futures Research Institute, Griffith University, Brisbane, Queensland 4111, Australia

${ }^{10}$ Department of Organismic and Evolutionary Biology, Harvard University, Cambridge, MA, 02138, USA

25 Corresponding author: ssegar@harper-adams.ac.uk (Simon T. Segar)

Twitter Handle: @simonsegar

Address: Department of Crop and Environment Sciences, Harper Adams University, Newport, Shropshire, TF10 8NB

Telephone: +441952815478

Key words: adaptive network, ecoevolution, metacommunity, microevolution, ecophylogenetics, phylogeny. 


\section{Abstract}

The structure of ecological networks reflects the evolutionary history of their biotic components, and their dynamics are strongly driven by ecoevolutionary processes. Here we present an appraisal of recent relevant research, in which the pervasive role of evolution within

45 ecological networks is manifest. Although evolutionary processes are most evident at macroevolutionary scales, they are also important drivers of local network structure and dynamics. We propose components of a blueprint for further research, emphasizing processbased models, experimental evolution and phenotypic variation, across a range of distinct spatial and temporal scales. Evolutionary dimensions are required to advance our understanding of foundational properties of community assembly and to enhance our capability of predicting how networks will respond to impending changes. 
65 Linking evolution and ecological networks across multiple levels

There is growing interest in the role of evolutionary history in determining how pairs of species interact [1]. At fine spatial scales, the evolution of one species is impacted directly by others through biotic natural selection and sometimes through reciprocal coevolution (see Glossary). Interacting species, however, do not persist as isolated pairs in time and space: they are

70 embedded within ecological networks involving multiple positive and negative interactions. Such a network perspective is essential for understanding the arena within which species interact. Network structure itself feeds back to influence evolutionary outcomes [2], and ongoing trait evolution [3]. Here we review the impact of evolution on ecological networks, taking a novel multiscale approach. We present a synthesis of previous work on the, often

75 interlinked, drivers of network structure across space (local to regional) and time (ecological to evolutionary) (Figure 1). While we appraise how an integrative and process-based approach will advance the understanding of network assembly and structure, we focus on how this has emerged from previous work through identifying current challenges and future directions.

\section{Evolutionary patterns in largescale networks}

\section{Macroevolution and phylogeography}

The availability of phylogenetic trees for entire clades allows identification of largescale phylogenetic patterns within networks [4]. Large datasets from sequencing studies are providing the taxonomic breadth required to connect networks with macroevolutionary studies of diversification [5,6]. Many networks are phylogenetically [7] and spatially

85 structured, shaped by both historical and ongoing selection [8] which may lead to topological convergence over evolutionary time (Box 1) $[9,10]$. For example, rapid adaptive radiations on islands produce networks of endemics rather than recurrent and recent invaders reflecting niche differentiation among close relatives, whereas sequential sympatric speciation on novel plant 
hosts can drive modularity in networks [11]. Network structure is dynamic through evolutionary time (Figure 1). In island chronosequences, older islands exhibit more modular networks, reflecting longer periods of in situ diversification [12]. Accordingly, a phylogeographic approach to network assembly is required, focusing on the distribution and evolution of species and their interactions across regions [13]. This will afford a greater understanding of pattern in ecology, as well as the underlying evolution of morphological,

95 behavioural and ecological traits.

\section{Phylogenetic network signals}

Phylogenetic patterns in networks are mediated by species-specific traits. As biotic [14] and abiotic conditions change so traits are modified, in turn producing network-level responses (Box 2). If traits are conserved within phylogenies they will act as proxies for phenotypic similarity by shared descent. Closely related species will often interact with sets of similarly closely related species [15] through niche conservatism (Figure 2). Strong ecophylogenetic patterns in extinction cascades and compartmentalisation of networks have been identified $[7,16,17]$, indicating the network-structuring roles of conserved traits.

Under the assumption of niche conservatism, phylogeny should help predict both species interactions and overall network topology [18]. For example, traits with a phylogenetic signal (such as flower symmetry and pollinator size) accurately predict interactions in some plantpollinator networks [19]. However, deviations from phylogenetic conservatism may occur (for instance via convergence) [20], suggesting that phylogenies should be combined with trait measurements [17,19].

\section{0 \\ Trait evolution}

To investigate the ecological influence of biotic drivers on trait evolution and network structure [21] we need to encompass ecological interactions and phenotypic evolution into phylogenetic 
comparative methods. Drury et al. [22] included competition when tracing the phylogenetic evolution of traits in Anolis lizards. Extending such an approach to predation, mutualism, and facilitation would help elucidate the assembly of interaction networks. Ancestral trait reconstruction may facilitate the modelling of trait transitions over time and, accordingly, the likelihood of interactions among species and lineages [23].

Although evolutionary processes within networks can lead to predictable structures [9], scaling microevolution in networks to macroevolutionary processes across phylogenies remains a challenge $[5,24]$. Intergenerational studies of phenotypic ecoevolution in one or two species [25] must be connected to trait evolution and processes such as speciation and extinction [2628]. Widely used neutral models of trait evolution (e.g. the Brownian motion model) can be extended to include processes such as stabilising selection, which generates a weak phylogenetic signal [20]. The parameterisation of relatively simple models of trait evolution with ecologically relevant data (e.g. heritability, effective population size and selection) across networks of species and across scales, remains a major challenge [29]. Extending the phylogenetic comparative approach is essential for exploring trait evolution in networks.

\section{Codiversification and coevolution}

Congruence between network and phylogenetic tree structures likely depends on the type and intimacy of interactions (e.g. mutualistic or antagonistic; obligate or facultative). Phylogenetic patterns of two connected trophic levels may be asymmetric or reciprocal. Asymmetrical phylogenetic patterns in networks [30] may result from phylogenetic tracking rather than reciprocal coevolution [31]. Furthermore, even when network patterns reflect phylogeny, reciprocal coevolution may not always be the driver. Congruence in phylogenetic branching among trophic levels may simply reflect biogeographic vicariance. Moreover, coevolution does not always produce trait and network correlations [32-34]. 
Comparing different network types provides strong evidence for evolution in networks. Phenotypic matching among interacting species leads to more modular networks and cycles of increased specialisation. In contrast, nested structures arise when interactions are mediated by phenotypic differences, particularly in mutualistic networks when coevolutionary selection is weak [35]. Food webs (antagonistic trophic networks) are often modular [36] with a strong phylogenetic signal [30]. Mutualistic pollination and dispersal networks are often nested [36], with fewer, frequently asymmetric, phylogenetically-clustered interactions [30] often with convergent traits. These patterns conform with the predictions of coevolutionary theory derived from pairwise and small group interactions [30,37].

Classically, antagonistic coevolution leads to an 'arms race' with escalation of defensive contra offensive phenotypes. This can lead to modular networks [38], particularly at larger phylogenetic scales. For example, Brassicaceous plants and pierid butterflies have undergone cyclical escalations of defence and detoxification traits, driving butterfly host-specificity [39]. Such an outcome is most likely in highly modular networks [40]: indeed, increased selection pressure on lower trophic levels and specialisation [31] of higher trophic levels favour the emergence of modularity. Additional mechanisms, such as coevolutionary alternation (where predator-prey preferences fluctuate through time) are also likely to influence network structure over longer timescales [41].

155 Coevolution can follow multiple alternative trajectories in mutualistic and antagonistic networks, influenced by and influencing network structure and evolutionary feedbacks [3,35]. When many specialist species are reliant on a few persistent generalist species (as in nested networks), coevolution in mutualistic networks may lead to increased resilience [42], connectance and 'coevolutionary rescue' through rewiring [35]. Within a single network type, 160 distinct structures can arise from differing evolutionary processes. Using simulations, Braga et al. [43] found that adaptive radiation in butterflies following a host-shift led to modular 
networks, whereas nested networks only arose during periods of expansion in host range. This combination of theoretically derived predictions with directed empirical studies is pivotal in the comparative approach we advocate. Since network structure is itself a "meta-trait", a crosscommunity approach provides a perspective for understanding such structures as outcomes of particular selective processes [44].

The clearest examples of phylogenetic pattern in networks result from codiversification among clades of interacting species. This is seen in entire clades of insects that are restricted to a particular clade of plants. Such strong signals in entire lineages in networks imply underlying coevolutionary processes. An extreme example is that of figs (Moraceae) and their pollinating wasps (Agaonidae). Genera of mutualistic, pollinating wasps and figs display phenotypic matching and congruence in clade ages. In contrast, networks of parasitic wasps derived from only distantly related clades invaded the fig-pollinator mutualism following phenotypic convergence, exhibiting more limited coevolution [45]. Phylogenetic tracking is likely to be more prevalent than codiversification, which is evident in the mismatch of phylogenetic timescales among trophic levels. This reflects the fact that ecological processes including network assembly may change over relatively short temporal and spatial scales, whereas macroevolutionary patterns emerge over much larger scales.

\section{Phylogenetic constraints and adaptive rewiring in networks}

Linking microevolution to macroevolutionary theory is central to the integration of network analyses across scales (see "Trait evolution"). Explicit inclusion of trait evolution using phylogenetic comparative methods is essential because traits are the outcome of macroevolution that determine current species interactions [21]. Modelling networks as adaptive landscapes is one way of linking phenotype, measures of fitness and network structure (Box 1). Adaptive networks [46] model the feedbacks between the states of each node and the 
topology of the network. By integrating ecoevolutionary, metacommunity [47] and geographic theories, adaptive network models can connect population and community-level processes (Box 1) [48]. Interactions are modulated by species-specific abundance dynamics and traits and may feed back to modify network structure [49]. Incorporating phylogeny into adaptive network models can improve predictions of network 'rewiring' in response to perturbation, if species interactions evince phylogenetic signals [48]. Incorporating traits and their variance with phylogeny can tease apart the relative contribution of phylogenetic constraints (when certain traits and interactions cannot occur due to the phylogenetic history of the interacting clades), ecological fitting and evolution in determining this adaptive rewiring. Modelling networks as evolving entities becomes possible through incorporating the mechanisms by which interactions themselves evolve [50] and are inherited as speciation proceeds.

\section{Phylogenetic determinants of network structure across different spatial scales}

The evolutionary history of interacting lineages filters regional species pools. For example, specialised natural enemies may be capable of suppressing range expansion of their hosts but not of invasive species. In an elegant laboratory experiment Carrasco et al. [51] demonstrated the impacts of separate or concurrent arrival of an invasive herbivore (Spodoptera littoralis) and its associated parasitoid (Microplitis rufiventris) on a native herbivore (Mamestra brassicae) and its parasitoid (Microplitis mediator) in determining fitness costs within a tritrophic network. Microplitis rufiventris varied in its negative impact on both native species according to the presence of Spodoptera littoralis. A second level of filtering by ecological processes sifts phylogenetically determined regional species pools into local networks of interacting species (Figures 2 and 3). The local species pool constrains realised interactions, but is itself constrained by proximal processes such as biogeography, physiological traits and 
interspecies dependencies superimposed on evolutionary history. A key task, therefore, is to elucidate and predict how local pools assemble (Figure 2). Habitat filtering can lead to phylogenetic clustering, whereas competition can lead to overdispersion. However,

215 interactions and ecological processes cannot be inferred solely from phylogenetic patterns [52] because assumptions of trait conservatism are not always met, and species interactions need not persist over evolutionary time [53].

\section{Regional species pools and meta-networks}

Most existing phylogenetic studies of local networks comprise incomplete, taxonomically

unbalanced subsets of diversity. Only sampling over wider spatial and phylogenetic scales will document regional pools of potential interactions from which locally recorded examples are drawn (see "Macroevolution and phylogeography"). Moreover, potential 'meta-networks' (Figure 1) constructed mechanistically from the total species pool (Figure 2) [54] allow us to model assembly through processes such as dispersal and extinction. Such modelled metanetworks are templates from which local networks can be extracted, conditional on evolutionary history and phenotypic matching. They also enable predictions of which species will not interact. In this way, inclusion of coevolution, ecological processes and life history traits into network hypotheses can take account of incompatibilities and 'forbidden links' [55].

Phylogenetic patterns within networks can change with downscaling from regional to local species pools as sets of 'available' species diminish, and interactions vary with local conditions (Figure 2). In other words, evolutionary history may predominate in shaping regional pools but will be harder to detect in local networks. Ponisio and M'Gonigle [33] modelled both coevolving and non-coevolving networks, and concluded that the topological legacy of coevolution within networks does not always reflect interaction intimacy. Increased knowledge of biogeographic ranges of species, as well as their interactions and phylogenetic histories, will 
enable assessment of the relative contributions of evolutionary and ecological conditions to observed network structures.

\section{Realised networks and ecoevolutionary feedbacks}

\section{Microevolution}

Two advances have fuelled understanding of ecoevolutionary feedbacks within networks. First, coevolution is now broadly accepted as a selective force comparable in importance to the abiotic environment [56]. Second, we can model phenotypic evolution in multiple interacting

245 species over ecological timescales $[57,58]$. Studies that quantify genotypic frequencies, phenotypic change and resultant interaction strengths, in the short term, have resulted in compelling evidence for this ecoevolutionary feedback [57,59]. Advances in modelling evolution in networks have not only generated hypotheses for empiricists but facilitate better connections across spatial and temporal scales (Box 1).

\section{Outcomes of ecoevolutionary feedbacks}

Ecoevolutionary feedbacks link simple systems with few components to complex networks $[60,61]$. Evolution among ecologically linked genotypes can influence emergent network properties propagating selective pressures on other species within the network. This should be observable in natural systems, particularly those susceptible to rapid genetic change (e.g. with 255 short-lived species and/or strong selective pressures).

Microcosm and mesocosm experiments involving short-lived organisms, especially microbes, provide a long-overdue integration of experimental evolution and network ecology informing models of network assembly and dynamics [62]. Microcosms are testing grounds for theoretical predictions by, for example, confirming that resistance genes can structure entire communities 260 [63] and are potentially important drivers of coevolutionary dynamics in networks [56]. 
Modelling these in a network superstructure suggests that evolution can lead to increased diversity and stability of networks [64]. Diversity likely insures ecosystem function at larger scales [65], although a general rule for diversity-stability relationships remains elusive [66]. Linking phenotypic adaptation with genotypic evolutionary change is challenging because the exact genetic control of most phenotypic traits is unknown. Most current models consider quantitative traits linking individual genotypes to phenotypic population means $[9,66,67]$, subsequently scaled up to include multiple traits and more realistic bounds on trait evolution $[68,69]$. Developments in whole-genome sequencing, transcriptomics and gene editing also bridge this gap. For example, gene editing in Drosophila melanogaster has indicated the evolutionary pathway leading to cardiac glycoside resistance in Danaus plexippus [70]. Resistance to host defences is a key predictor of host use in food webs. A marriage of comparative and experimental approaches will bridge the micro-macroevolutionary divide [71].

Ecoevolutionary feedbacks in spatial networks

Ecoevolutionary feedbacks in ecological networks will operate spatially as well as temporally. Both ecological and microevolutionary processes show strong spatial dependence. Spatial heterogeneity in abiotic conditions results in species sorting within metacommunities, with consequent variation in the relative fitness of genotypes [72]. Dispersal of individuals among

280 local networks in a metacommunity can affect both the species composition of networks (since individuals represent potential colonists) as well as the ability of species to adapt to local conditions (because colonists carry alleles that could either facilitate or swamp local adaptations). These spatial effects can have important ecoevolutionary feedbacks, within socalled "evolving metacommunities" [47]. Early colonization of a habitat leading to rapid local 285 adaptation may exclude subsequent colonists: an ecoevolutionary priority effect $[73,74]$. 
The geographic mosaic theory of coevolution predicts that the genotypes of coevolving species will be impacted as species interactions are moulded by environmental heterogeneity [37]. In consequence, interaction strengths and species persistence in local networks may change [75]. In partial support of this prediction, environmental gradients that correlate with genotype 290 change in host plants have been shown to affect interaction strengths within associated arthropod networks [76] as well as species composition [77]. Full integration of ecoevolutionary feedbacks into spatially structured and speciose networks is a problem of high dimensionality and, in the short term, empirical progress will likely focus on the "hub" species that connect local networks in space [75].

\section{Intraspecific variation}

We have increasing understanding of how genes coding for phenotypic traits affect fitness outcomes within interactions, and how intraspecific variation contributes to network persistence [78]. Incorporating genotypic diversity into empirical and simulation studies will help us to understand how networks themselves evolve and persist [79]. For example, plant genotypes can determine the level of resistance to herbivory, affecting networks of insect herbivores and even their parasitoids [80]. Linking the genotypic composition of interacting species and, thereby, identifying networks of interacting genes across species via gene expression, allows genotypic change to be studied in the same detail as population change [39].

Hitherto such studies have focused on pairwise interactions. By scaling up to networks, we can explore whether genotypes are important predictors of multispecies interactions and network structure (Figure 3) and, conversely, which biotic interactions generate and are affected by genotypic diversity. This is now central to understanding how evolutionary processes permeate and reflect network configurations. Phenotypic evolution also can be mediated by ecological 

phenotypes can also be modelled along the branches of phylogenetic trees under fluctuating ecological conditions. This continuum provides a natural connection to macroevolutionary temporal and spatial scales.

\section{Prospects for tighter cohesion across network levels}

Multi-layered networks

A significant step will be to build ecoevolutionary models that span a range of levels across the biological hierarchy. We can now model how gene interaction networks (i.e. evolutionary networks) predict phenotypic trait variation which, in turn, determines interactions within networks [81]. The next step is to expand the focus to encompass multi-layer networks. Promising approaches include the integration of different network layers through shared nodes, where layers represent different spatial or temporal dimensions or interaction modes [82,83] (Figure 1). An example involving diverse interaction modes would be a network comprising layers for plants, their antagonistic insect herbivores, and their mutualistic pollinators [84].

325 Adding a spatial dimension can be achieved by linking interactions occurring in a habitat patch to those occurring within wider landscapes [85]. Intersections of these spatial scales offer prospects for exciting future work which will elucidate how changes in phenotypes cascade up to the regional species pool.

\section{Concluding remarks and future perspectives}

Emergent properties of complex networks of interacting species are a result of evolution. Interactions within any given network are embedded in large and complex networks of networks. Therefore, a larger spatio-temporal scale is required to understand fully the evolution 
of the regional species pool, the raw material for all such networks. Evolutionary change, often acting through biotic interactions, shapes phenotypic traits and, in turn, influences future species interactions. Simultaneously, trait trajectories are further modified by reciprocal selection among networks. Macroevolutionary change is most easily linked to phylogenies using the phylogenetic comparative method, which provides a tractable approach for detecting the signature of evolution at regional scales [24]. Three major research developments are 340 helping to link networks with microevolutionary processes:

i) Increasingly realistic, process-based models of indirect coevolution that link network layers across evolutionary and spatial scales.

ii) Studying evolution in networks across ecological scales in tractable experimental systems that enable better separation of cause and effect.

345 iii) An explicit inclusion of phenotypic variation as the raw material on which selection acts within networks to maximise fitness, for example adaptive models of network assembly that consider variation in individual trait values and species' abundances (see Outstanding Questions).

350 We propose that the linkage of evolutionary processes and their ecological consequences across spatial and temporal scales is an obligate component of future research. Progress in this area will be achieved by exploring selective landscapes and their inherent fluidity, facilitated by individual-level adaptive simulations of network assembly. Ecoevolutionary feedback can be incorporated into network studies by mapping traits onto phylogenies. A major focus of new research should be modelling heritable trait changes within networks, between generations or after speciation events, permitting prediction of interactions and network topology [24]. By modelling using multiple real-world parameter sets, realistic trait values and variable interaction types we can simulate sets of interconnected [84] meta-networks, allowing them to 
undergo in silico 'selection' together with periodic rewiring based on realistic levels of perturbation [48]. This process-based approach will produce insights into species coexistence and diversity-stability relationships. Synthetic (and empirical) networks can then be compared in a likelihood framework [23] as sets of alternatives, themselves part of a wider pool of selective regimes [81]. Adding the requisite spatial dynamics can be achieved by allowing transfer of individuals between networks $[47,86]$, so that transitions across scales will become 365 part of a natural continuum. Network structure itself will inform the probability of persistent interactions in subsequent generations. Such an integrative approach will provide incentive for empirical ecologists to consider intraspecific variation (and its genetic basis) as an additional parameter for predicting interaction matrices and trait values within networks. Studies of experimental evolution in networks will gain a powerful predictive approach and unified 370 framework.

Networks lie at the heart of ecosystems, therefore the advances we outline and envisage will enhance ecology as a predictive discipline. Through connecting the evolution of speciesinteraction networks to the most foundational issue in ecology - the recognition and understanding of pattern across multiple scales - we expect that a more complete understanding 375 of fundamental processes in community ecology will emerge. For example, the processes of community assembly and succession are driven by strong ecoevolutionary and spatial components played out across networks of networks. On a larger scale, species pools and standing diversity are greatly influenced by biotic selection over evolutionary time.

Ecological networks, when viewed as the coincident products of sets of evolutionary processes, are necessarily dynamic: as their selective environment responds to change, so will the configuration of the network. Adoption of a network framework will inevitably benefit our understanding of trait evolution itself and, at the other extreme, of ecosystem level responses to perturbations. Predictions of how networks will be rewired as a result of environmental, 
especially human-driven changes (such as species introductions and their local adaptation) must take evolutionary relationships and phenotypic evolution into account [42]. The stability of local networks will, inter alia, reflect the degree of coevolution among participating species. Understanding and predicting how evolutionary processes interact with network structuring and persistence will play a major role in understanding and managing such changes.

390

395 


\section{Glossary}

"A network whose links change adaptively with respect to its states, resulting in a dynamical interplay between the state and the topology of the network" [46].

\section{Antagonistic networks}

Networks in which the links represent interactions with negative impacts on the fitness of one

415 level of interacting species.

\section{Brownian motion}

The evolution of a continuous trait across a phylogeny, modelled as a random walk for comparison with other processes [20].

\section{Coevolution}

420 The mutual and concurrent evolutionary adaptation of traits in a population of one species to individuals from another. [34].

\section{Codiversification}

The simultaneous diversification (speciation) of two interacting lineages.

\section{Community phylogenies}

425 Phylogenies pruned to include only cooccurring species rather than all species within a taxon or clade.

\section{Ecoevolutionary feedback}

"The cyclical interaction between ecology and evolution such that changes in ecological interactions drive evolutionary change in organismal traits that, in turn, alter the form of ecological interactions, and so forth" [87] 


\section{Ecological fitting}

"The process whereby organisms colonize and persist in novel environments, use novel resources or form novel associations with other species as a result of the suites of traits that they carry at the time they encounter the novel condition" [88].

\section{Ecological network}

Any depiction of a set of interindividual or interspecies interactions in nature, usually comprising nodes (the species themselves) and edges (the functional links among species).

\section{Ecophylogenetics}

A field of study focused on exploring patterns and process in ecology through the 440 combination of ecological data with phylogenetic and biogeographic data.

\section{Interaction strength}

The frequency and/or fidelity of a connection between two nodes in a network when sampled at multiple points (across time and/or space).

\section{Microevolution}

445 Microevolution relates specifically to the turnover of allele frequencies within a population through inheritance, selection and drift.

\section{Macroevolution}

Evolution on a scale at or above the level of species.

\section{Mutualistic networks}

450 Networks in which the links represent interactions with positive impacts on the fitness of both sets of interacting species. 


\section{Network phylogenetic signal}

The statistical nonindependence, and phylogenetic clustering, among interactions in a network due to the phylogenetic relatedness of nodes (modified from [20]).

\section{Network stability}

A multidimensional component (metrics include persistence, robustness, resistance, resilience and variability) that quantifies the ability of a network to resist restructuring following perturbation.

\section{Niche conservatism}

460 The tendency of species to retain ancestral traits.

\section{Phenotypic plasticity}

"The ability of individual genotypes to produce different phenotypes when exposed to different environmental conditions" [89].

\section{Phenotypic matching}

465 Phenotypic resource traits that match those of consumers, e.g. phenological cooccurrence of plants and pollinators.

\section{Phenotypic differences}

The extent to which a trait in one species exceeds or overcomes a corresponding trait in another (e.g. animal gape must exceed fruit diameter in seed dispersal mutualisms) [35].

\section{$470 \quad$ Phylogenetic tracking}

"Phylogenetic tracking occurs if there is strong asymmetry in the interaction between two species, implying one species is much more dependent on the other. This leads to parallel phylogenetic trees" [31]. 


\section{Trait}

475 Morphological, behavioural, ecological or chemical features of a species reflecting both its evolutionary history and its local phenotypic adaptation. 


\section{Box 1: Modelling the evolutionary processes that determine interaction networks across}

spatial and evolutionary scales

Evolution has been incorporated into models of network self-assembly by allowing macroevolutionary change in nodes [90]. The resulting networks are architecturally similar to real networks [66]. Such models have now been extended to include multiple traits with more realistic bounds on trait evolution $[68,69]$. The trait evolution of a given species can be affected by interactions with multiple species. More complex, non-additive, selection can occur when there are indirect interactions. These different selection pressures can be integrated into network models, at least for single trait coevolution, and simulations have shown that indirect interactions can be major selective agents for trait changes in mutualisms [3].

Links within ecological networks are frequently represented as between species-level nodes, but in fact individual and population level traits determine interactions and their frequencies. Furthermore, traits and abundances fluctuate in time and space resulting in non-static networks [91]. For example, a widespread generalist may be comprised of many local specialists in different parts of its range. Dynamic network models [49] are predictive, in that they build up networks according to point variation in phenotypic traits, individual abundance and feedbacks among individuals (including indirect interactions). Since they are processbased, niche or neutral mechanisms can be modelled. Importantly, this approach bridges the gap between local and regional scales, enabling the comparison of networks on a biogeographic scale while still being sensitive to regional variation. Adaptive network models [46] provide a link between population level processes driving trait evolution and the

515 interactions that shape networks and communities. Evolving metacommunity models describe how ecoevolutionary feedbacks act across the selective landscape, resulting in changes in network organisation that alter the distribution of traits between interacting species in a reciprocal manner [47]. Inclusion of evolutionary history into dynamic models can help us to 
predict how species interactions may be rewired [48] and shed light on the processes shaping phylogenetic signal as a pattern in ecological networks. Fluctuations in phenotypic traits resulting from coevolution can also be integrated into dynamic and adaptive models, in combination with spatial variation in interaction strength. Metacommunity models facilitate the description of meta-networks and their derived local networks. Combining the metacommunity models [92] used to describe meta-networks and local networks with

525 adaptive network models and community phylogenies will be important in linking processes across spatial and evolutionary scales.

\section{Box 2: Evolutionary history as a predictor of network responses to human impacts}

The impacts of novel environmental gradients generated by humans on species composition are now relatively well-known, and their effects on interactions between species are becoming apparent [93]. However, the influence of evolutionary history on network responses to global changes, the degree to which networks can adapt to global changes through evolution, and subsequent longer-term consequences for evolution of networks remain mostly unknown [but see 94].

Network structure can predict impacts of cascading extinctions [95] or identify keystone species. Most simply, phylogenetic relatedness has been incorporated into models simulating the impacts of species extinctions on networks. If there are phylogenetic signals in the network then cascading extinctions are predicted to lead to non-random loss of species from phylogenies, with greater risk of entire clades going extinct [18]. For example, pollination networks in smaller and less isolated forest fragments show a lower degree of phylogenetic

540 matching, presumably owing to extinction of more specialized species and invasion by generalists [7]. This is of concern because conservationists often assign high value to phylogenetic diversity and hence prioritise to reduce its loss [96]. Furthermore, the effects of 
biodiversity on ecosystem functioning depend critically on how species reorganize within networks following species loss [97].

545 An important next step in understanding species' responses to global changes will be to incorporate traits into such analyses. This approach is already being used regarding individual species' responses to global changes, with impacts of multiple traits on fitness of species in new abiotic environments being calculated to describe "dynamic adaptive landscapes" [98]. This approach could be profitably extended to novel biotic assemblages, by quantifying fitness changes in a focal species due to shifts in its inclusive network.

An additional complication is that species may evolve in response to altered biotic and abiotic environments [99]. However, it is often challenging to determine whether observed changes are due to existing phenotypic plasticity, or to changes in genotypes [100]. For example, mutualists may shift to become more antagonistic, or even abandon interactions completely 555 [94]. If the fitness outcomes of being involved in an interaction shift, though not to the extent that species go extinct in the short term, then we would expect them to adapt to their novel biotic environment. 
STS was funded by GACR grant 15-24571S, he is grateful for departmental support from Harper Adams University. TMF was funded by a GACR grant 19-14620S. OTL was funded by the Natural Environment Research Council (NE/N010221/1). TML received support from FAPESP (Grant 2016-24821-1), CNPq (Grant 311800/2015-7) and an EURIAS Fellowship 570 Grant at the Wissenschaftskolleg zu Berlin. VN was funded by GACR grant 17-23862S. We thank Andrea Stephens, five anonymous reviewers, Jane Memmott, Stuart Pimm, Conor Redmond and Daniel Souto-Vilaros for commenting on various versions of this manuscript.

\section{References}

1 Mouquet, N. et al. (2012) Ecophylogenetics: advances and perspectives. Biol. Rev. 87, 769-785

5752 de Andreazzi, C.S. et al. (2018) Eco-evolutionary feedbacks promote fluctuating selection and long-term stability of antagonistic networks. Proc. R. Soc. B: Biol. Sci. 285, 20172596

3 Guimarães, P.R. et al. (2017) Indirect effects drive coevolution in mutualistic networks. Nature 550, 511-514

4 Ponisio, L.C. et al. (2019) A network perspective for community assembly. FEVO 7, 103

5805 Weber, M.G. et al. (2017) Evolution in a community context: on integrating ecological interactions and macroevolution. Trends Ecol. Evol. 32, 291-304

6 Hinchliff, C.E. et al. (2015) Synthesis of phylogeny and taxonomy into a comprehensive tree of life. Proc. Natl Acad. Sci. USA 112, 6

7 Aizen, M.A. et al. (2016) The phylogenetic structure of plant-pollinator networks increases with

8 Peralta, G. et al. (2015) Phylogenetic diversity and co-evolutionary signals among trophic levels change across a habitat edge. Journ. Anim. Ecol. 84, 364-372

9 Loeuille, N. and Loreau, M. (2005) Evolutionary emergence of size-structured food webs. Proc. Natl Acad. Sci. USA 102, 5761-5766

59010 Pascual, M. and Dunne, J.A., eds. (2005) Ecological Networks: Linking Structure to Dynamics in Food Webs, Oxford University Press.

11 Forbes, A.A. et al. (2009) Sequential sympatric speciation across trophic levels. Science 323, 776-779

12 Rominger, A.J. et al. (2016) Community assembly on isolated islands: macroecology meets evolution. Glob. Ecol. Biogeogr. 25, 769-780

13 Bunnefeld, L. et al. (2018) Whole-genome data reveal the complex history of a diverse ecological community. Proc. Natl Acad. Sci. USA 115, E6507-E6515

14 Pearse, I.S. and Altermatt, F. (2013) Predicting novel trophic interactions in a non-native world. Ecol. Lett. 16, 1088-1094

60015 Ives, A.R. and Helmus, M.R. (2011) Generalized linear mixed models for phylogenetic analyses of community structure. Ecol. Monogr. 81, 511-525

16 Rezende, E.L. et al. (2007) Effects of phenotypic complementarity and phylogeny on the nested structure of mutualistic networks. Oikos 116, 1919-1929

17 Rezende, E.L. et al. (2009) Compartments in a marine food web associated with phylogeny, 
18 Rezende, E.L. et al. (2007) Non-random coextinctions in phylogenetically structured mutualistic networks. Nature 448, 925-928

19 Chamberlain, S.A. et al. (2014) Traits and phylogenetic history contribute to network structure across Canadian plant-pollinator communities. Oecologia 176, 545-556

61020 Revell, L.J. et al. (2008) Phylogenetic signal, evolutionary process, and rate. Syst. Biol. 57, 591601

21 Pennell, M.W. and Harmon, L.J. (2013) An integrative view of phylogenetic comparative methods: connections to population genetics, community ecology, and paleobiology. Ann. $N Y$ Acad. Sci. 1289, 90-105

61522 Drury, J. et al. (2016) Estimating the effect of competition on trait evolution using maximum likelihood inference. Syst. Biol. 65, 700-710

23 Manceau, M. et al. (2017) A unifying comparative phylogenetic framework including traits coevolving across interacting lineages. Syst. Biol. 66, 18

24 Harmon, L.J. et al. (2019) Detecting the macroevolutionary signal of species interactions. J.

620 Evol. Biol. 32, 769-782

25 Schoener, T.W. (2011) The newest synthesis: understanding the interplay of evolutionary and ecological dynamics. Science 331, 426-429

26 Arnold, S.J. et al. (2001) The adaptive landscape as a conceptual bridge between micro- and macroevolution. Genetica 112, 9-32

62527 Arnold, S.J. (2014) Phenotypic evolution: the ongoing synthesis: (American Society of Naturalists address). Am. Nat. 183, 729-746

28 Uyeda, J.C. et al. (2011) The million-year wait for macroevolutionary bursts. Proc. Natl Acad. Sci. USA 108, 15908-15913

29 Uyeda, J.C. and Harmon, L.J. (2014) A novel bayesian method for inferring and interpreting the dynamics of adaptive landscapes from phylogenetic comparative data. Syst. Biol. 63, 902-918

30 Rohr, R.P. and Bascompte, J. (2014) Components of phylogenetic signal in antagonistic and mutualistic networks. Am. Nat. 184, 556-564

31 Russo, L. et al. (2018) Quantitative evolutionary patterns in bipartite networks: Vicariance, phylogenetic tracking or diffuse co-evolution? Methods Ecol. Evol. 9, 761-772

63532 Nuismer, S.L. et al. (2010) When is correlation coevolution? Am. Nat. 175, 525-537

33 Ponisio, L.C. and M'Gonigle, L.K. (2017) Coevolution leaves a weak signal on ecological networks. Ecosphere 8, e01798

34 Janzen, D.H. (1980) When is it coevolution? Evolution 34, 611-612

35 Nuismer, S.L. et al. (2013) Coevolution and the architecture of mutualistic networks: coevolving networks. Evolution 67, 338-354

640

36 Thébault, E. and Fontaine, C. (2010) Stability of ecological communities and the architecture of mutualistic and trophic networks. Science 329, 853

37 Thompson, J.N. (1994) The Coevolutionary Process, The University of Chicago Press.

38 Beckett, S.J. and Williams, H.T.P. (2013) Coevolutionary diversification creates nested-modular structure in phage-bacteria interaction networks. Interface Focus 3, 20130033-20130033

39 Edger, P.P. et al. (2015) The butterfly plant arms-race escalated by gene and genome duplications. Proc. Natl. Acad. Sci. USA 112, 8362-8366

40 Andreazzi, C.S. et al. (2017) Network structure and selection asymmetry drive coevolution in species-rich antagonistic interactions. Am. Nat. 190, 99-115

65041 Nuismer, S.L. and Thompson, J.N. (2006) Coevolutionary alternation in antagonistic interactions. Evolution 60, 2207-2217

42 Nuismer, S.L. et al. (2018) Coevolution slows the disassembly of mutualistic networks. Am. Nat. 192, 490-502

43 Braga, M.P. et al. (2018) Unifying host-associated diversification processes using butterflyplant networks. Nat. Commun. 9, 5155 
44 Borrelli, J.J. et al. (2015) Selection on stability across ecological scales. Trends Ecol. Evol. 30, 417-425

45 Segar, S.T. et al. (2013) Convergent structure of multitrophic communities over three continents. Ecol. Lett. 16, 1436-1445

66046 Gross, T. and Sayama, H. (2009) Adaptive networks. In Adaptive networks pp. 1-8, Springer

47 Urban, M. et al. (2008) The evolutionary ecology of metacommunities. Trends Ecol. Evol. 23, 311-317

48 Raimundo, R.L.G. et al. (2018) Adaptive networks for restoration ecology. Trends Ecol. Evol. 33, 664-675

66549 Poisot, T. et al. (2015) Beyond species: why ecological interaction networks vary through space and time. Oikos 124, 243-251

50 Poisot, T. and Stouffer, D.B. (2016) How ecological networks evolve, Ecology.

51 Carrasco, D. et al. (2018) With or without you: Effects of the concurrent range expansion of an herbivore and its natural enemy on native species interactions. Glob. Chang. Biol. 24, 631-643

670

52 Godoy, O. et al. (2014) Phylogenetic relatedness and the determinants of competitive outcomes. Ecol. Lett. 17, 836-844

53 Mayfield, M.M. and Levine, J.M. (2010) Opposing effects of competitive exclusion on the phylogenetic structure of communities: Phylogeny and coexistence. Ecol. Lett. 13, 1085-1093

54 Dunne, J.A. (2006) The network structure of food webs. In Ecological Networks: Linking

675 Structure to Dynamics in Food Webs pp. 27-86, Oxford University Press

55 Strona, G. and Veech, J.A. (2017) Forbidden versus permitted interactions: Disentangling processes from patterns in ecological network analysis. Ecol. Evol. 7, 5476-5481

56 Brockhurst, M.A. and Koskella, B. (2013) Experimental coevolution of species interactions. Trends Ecol. Evol. 28, 367-375

57 DeLong, J.P. et al. (2016) How fast is fast? Eco-evolutionary dynamics and rates of change in populations and phenotypes. Ecol. Evol. 6, 573-581

58 Govaert, L. et al. (2019) Eco-evolutionary feedbacks - Theoretical models and perspectives. Funct. Ecol. 33, 13-30

59 Govaert, L. et al. (2016) Eco-evolutionary partitioning metrics: assessing the importance of ecological and evolutionary contributions to population and community change. Ecol. Lett. 19, 839-853

60 De Meester, L. et al. (2018) Analyzing eco-evolutionary dynamics - the challenging complexity of the real world. Funct. Ecol. 33, 43- 59

61 terHorst, C.P. et al. (2018) Evolution in a community context: trait responses to multiple species interactions. Am. Nat. 191, 368-380

62 Weitz, J.S. et al. (2013) Phage-bacteria infection networks. Trends Microbiol. 21, 82-91

63 Bohannan, B.J.M. and Lenski, R.E. (2000) Linking genetic change to community evolution: insights from studies of bacteria and bacteriophage. Ecol. Model. 3, 362-377

64 Mougi, A. (2020) Natural selection contributes to food web stability. PLOS ONE 15, e0227420

69565 Yachi, S. and Loreau, M. (1999) Biodiversity and ecosystem productivity in a fluctuating environment: The insurance hypothesis. Proc. Natl. Acad. Sci. USA 96, 1463-1468

66 Loeuille, N. (2010) Influence of evolution on the stability of ecological communities. Ecol. Lett. $13,1536-1545$

67 Loeuille, N. and Loreau, M. (2009) Emergence of complex food web structure in community evolution models. In Community Ecology (Verhoef, H. A. and Morin, P. J., eds), pp. 163-178, Oxford University Press

68 Allhoff, K.T. et al. (2015) Evolutionary food web model based on body masses gives realistic networks with permanent species turnover. Sci. Rep. 5,

69 Ritterskamp, D. et al. (2016) A new dimension: Evolutionary food web dynamics in two dimensional trait space. J. Theor. Biol 405, 66-81 
70 Karageorgi, M. et al. (2019) Genome editing retraces the evolution of toxin resistance in the monarch butterfly. Nature DOI: 10.1038/s41586-019-1610-8

71 Weber, M.G. and Agrawal, A.A. (2012) Phylogeny, ecology, and the coupling of comparative and experimental approaches. Trends Ecol. Evol. 27, 394-403

71072 Gómez, J.M. and Perfectti, F. (2012) Fitness consequences of centrality in mutualistic individual-based networks. Proc. R. Soc. B: Biol. Sci. 279, 1754-1760

73 Loeuille, N. and Leibold, M.A. (2008) Evolution in metacommunities: on the relative importance of species sorting and monopolization in structuring communities. Am. Nat. 171, 788-799

71574 Urban, M.C. and De Meester, L. (2009) Community monopolization: local adaptation enhances priority effects in an evolving metacommunity. Proc. Roy. Soc. B Biol. Sci. 276, 4129-4138

75 Toju, H. et al. (2017) Species-rich networks and eco-evolutionary synthesis at the metacommunity level. Nat. Ecol. Evol. 1,

76 Toju, H. and Sota, T. (2006) Imbalance of predator and prey armament: geographic clines in phenotypic interface and natural selection. Am. Nat. 167, 105-117

77 Kagiya, S. et al. (2018) Does genomic variation in a foundation species predict arthropod community structure in a riparian forest? Mol. Ecol. 27, 1284-1295

78 Hart, S.P. et al. (2016) How variation between individuals affects species coexistence. Ecol. Lett. 19, 825-838

72579 Fortuna, M.A. et al. (2013) Evolving digital ecological networks. PLOS Comput. Biol. 9, e1002928

80 Barbour, M.A. et al. (2016) Genetic specificity of a plant-insect food web: Implications for linking genetic variation to network complexity. Proc. Natl Acad. Sci. USA 113, 2128-2133

81 Melián, C.J. et al. (2018) Deciphering the interdependence between ecological and evolutionary networks. Trends Ecol. Evol. 33, 504-512

82 Timóteo, S. et al. (2018) Multilayer networks reveal the spatial structure of seed-dispersal interactions across the Great Rift landscapes. Nat. Commun. 9,

83 García-Callejas, D. et al. (2018) Multiple interactions networks: towards more realistic descriptions of the web of life. Oikos 127, 5-22

73584 Pilosof, S. et al. (2017) The multilayer nature of ecological networks. Nat. Ecol. Evol. 1, 0101

85 Hackett, T.D. et al. (2019) Reshaping our understanding of species' roles in landscape-scale networks. Ecol. Lett. 22, 1367-1377

86 Nuismer, S.L. et al. (1999) Gene flow and geographically structured coevolution. Proc. R. Soc. B: Biol. Sci. 266, 605

74087 Post, D.M. and Palkovacs, E.P. (2009) Eco-evolutionary feedbacks in community and ecosystem ecology: interactions between the ecological theatre and the evolutionary play. Phil. Trans. Roy. Soc. B. 364, 1629-1640

88 Agosta, S.J. (2006) On ecological fitting, plant-insect associations, herbivore host shifts, and host plant selection. Oikos 114, 556-565

74589 Pigliucci, M. (2006) Phenotypic plasticity and evolution by genetic assimilation. J. Exp. Biol. 209, 2362-2367

90 Brännström, Å. et al. (2012) Modelling the ecology and evolution of communities: a review of past achievements, current efforts, and future promises. Evol. Ecol. Res. 14, 601-625

91 Poisot, T. et al. (2012) The dissimilarity of species interaction networks. Ecol. Lett. 15, 13531361

92 Urban, M.C. and Skelly, D.K. (2006) Evolving metacommunities: toward an evolutionary perspective on metacommunities. Ecology 87, 1616-1626

93 Tylianakis, J.M. et al. (2008) Global change and species interactions in terrestrial ecosystems. Ecol. Lett. 11, 1351-1363 
95 Dunne, J.A. and Williams, R.J. (2009) Cascading extinctions and community collapse in model food webs. Phil. Trans. Roy. Soc. B. 364, 1711-1723

96 Veron, S. et al. (2018) Predicting the impacts of co-extinctions on phylogenetic diversity in mutualistic networks. Biol. Conserv. 219, 161-171

76097 Reiss, J. et al. (2009) Emerging horizons in biodiversity and ecosystem functioning research. Trends Ecol. Evol. 24, 505-514

98 Laughlin, D.C. and Messier, J. (2015) Fitness of multidimensional phenotypes in dynamic adaptive landscapes. Trends Ecol. Evol. 30, 487-496

99 Agrawal, A.A. (2001) Phenotypic plasticity in the interactions and evolution of species. Science 294, 321-326

765

100 Franks, S.J. et al. (2014) Evolutionary and plastic responses to climate change in terrestrial plant populations. Evol. Appl. 7, 123-139 


\section{Figure Legends and Titles}

\section{5 \\ A conceptual framework for drivers of network structure across evolutionary and spatial scales}

Figure 1. The pool from which interacting species are drawn is shaped by macroevolutionary processes (e.g. speciation, adaptation and extinction) which are modified by species migration among regions as well as environmental and biotic heterogeneity. Here we summarise

800 important processes that lead to changes in network properties over evolutionary time (y axis) and with increasing spatial scale ( $\mathrm{x}$ axis). Additional links (edges) and species (nodes) are introduced to networks over both evolutionary time and with increasing spatial scale. Links and species are also lost as a result of species extinction over evolutionary time. The $\mathrm{x}$ axis shows how both local and regional scale networks are embedded within the global network.

805 These multi-layered networks are linked by nodes shared across time and across space.

\section{Pathways to network assembly I: from regional to local scales}

Figure 2. A hypothetical bipartite regional network (A) consisting of resource species (letters 810 in circles) and consumer species (numbers in circles). The evolution of a hypothetical continuous trait is illustrated for each trophic level. Optimal phenotypic matching occurs between producer and consumer traits, such that there is network phylogenetic signal and interactions are determined by phylogenetically conserved phenotypic matching. Processes such as environmental filtering (B) and competition (C) influence the draw of species from the 815 available pool, in turn affecting local network structure.

\section{Pathways to network assembly II: from the local scales to realised networks}


Figure 3. (A) Hypothetical changes in trait values based on a subset of interactions depicted in Figure 2. Trait values are represented by a normal distribution on the x-axis. Interactions are mediated by trait matching among trophic levels, such as that which may occur in a plant820 pollinator network, but trait values are modified as new species (with slightly different trait values) are added to the network. The arrival of a new consumer might select for modified traits in producers and the arrival of a resource might select for modified traits in consumers depending on the realised encounter frequency between consumers and producers, and on consumer preferences. (B) Phenotypes can be plastic, determined largely by the environment, 825 and/or have a strong genetic component. This scheme focuses on genetic determination. As selection acts on individuals within a population, the frequency of genotypes related to adaptive traits will change. In this case the arrival of a consumer (sp. 3) causes a genetically determined shift in producer phenotype (sp. B) and selection acts against genotype 1 of species B. Subsequent selection results in convergence or divergence in traits between consumers (sp. 2 830 and sp. 3) and a corresponding re-shuffle of genotype frequencies. In the final network module, a resource arrives (sp. C) that can be used only by consumer sp. 3, reducing competition between consumer species and modifying the selective landscape for sp. 2 . 